\title{
ADMISSION CONTROL FOR VIDEO TRAFFIC STREAMS WITH SCALING CHARACTERISTICS*
}

\section{Raniery Pontes and Rosângela Coelho}

\begin{abstract}
The impact of time-dependence or scaling characteristics on video acceptance regions is examined on this paper. We considered two different Call admission control (CAC) mechanisms; i) a descriptor-based CAC (DBCAC) mechanism and ii) a measurement-based CAC (MBCAC) mechanism. The proposed MBCAC is a hybrid measurement scheme that includes a Kalman filter and a real-time Hurst estimation hased on wavelets. We investigated several buffer sizes and video sequences with different dependence degrees. Furthermore, for the accuracy of the Hurst (H) estimation. we developed a Hurst Estimator Package (HEP). This package consists of the estimators: Rescaled adjusted range (R/S), Higuchi and Abry-Veitch wavelet (AV). An important result showed that video traffic connections with long-range dependence (LRD) and short-range dependence (SRD) have similar admission regions.
\end{abstract}

Keywords: Scaling or dependence characteristics, Hursi estimation. Video traffic. Fractional Brownian Motion. Admission Control.

Resumo - O impacto do grau de dependencia temporal nas regiōes de admissão de conexôes de vídeo é examinado neste trabalho. Dois mecanismos de controle de admissão de chamadas (Call admission control-CAC) foran analisados; (i) Mecanismo baseado em descritores de tráfego (DBCAC) e. (ii) Mecanismo baseado em medidas (MBCAC). O MBCAC proposto. possui um filıro de Kalman (para estimação da média e variância) e um estimador do parânetro de Hurst $(\mathrm{H})$ para uso em tempo real baseado en 11crielets. A análise foi realizada para diversas sequiências de vídeo com diferentes graus de dependência (Hurst) e tamanhos de buffer. Para a obtenção de uma estimação do grau de dependência nais precisa, foi desenvolvido um pacote denominado Hurst Estimator Package (HEP). Este pacote inclue os estimadores: Rescaled Adjusted range (R/S), Higuchi e Abry-Veitch wavelet(AV)). Un importante resultado deste trabalho demonstrou que conexões de vídeo com dependência de longo alcance (LRD-long-range dependence) e curto alcance (SRD-short-range dependence) possuem regiões de admissão similares.

Palavras-chave: Escala e Dependência Temporal. Estimação de Hurst. Trafego de Vídeo. Movimento browniano fracionário. Controle de admissão.

Raniery Pontes is currently with the Rede Nacional de Pesquisa (RNP). Rosângela Coelho is with the Electrical Engineering Department al the Instituto Militar de Engenharia do Rio de Janeiro (TME). E-mail; coelho (aime.el.br. Review coordinated by Nelson L.S. Fonseca (Area Ediror). Manuscript received Aug/13/2001. reriewed Dec/19/2001. aceepted Jan/04/2002.

\section{INTRODUCTION}

Call admission control of lime-dependent or scaling video connections is still an important issue for network traffic engineering. This dependence degree is expressed by the Hurst parameter. Video streams have an inherent timedependence due to the encoding process. The presence of time-dependence or scaling characteristics were observed on different traffic streams $[15,8,4,16,6]$. The performance of time-dependent traffic is a hot research topic specially when considering streams with long-range dependence or $H>1 / 2$. The strean dependence impact on buffer dimensioning and network performance is still under discussion [28.24] and the matter is not closed. In this paper. we examined the impact of the video connections scaling $(0<H<1)$ on admission regions.

As we are dealing with lime-dependent connections we need an accurate Hurst estimation to detcrmine the admission regions. Diffcrent estimation methods shall be applied to achieve this estimation accuracy. The methods proposed in the literature were developed considering different samples distributions and characteristics. For instance. the Higuchi method is better for fractal samples. The AV filtering procedure is not interesting for short samples sequences. i.e.. less than 1000. We developed a package named Hurst Estimator Package (HEP) consisting of the estimators, R/S [1 l]. Higuchi [29] and AV [27] (wavelet). The time-dependence analysis is then presented for these three estimators.

The CAC function determines the acceptance or rejection of a new connection and guarantees the required quality-ofservice (QoS) of all connections. We focused on CAC mechanisms based on the effective bandwidth (EB) decision criteria. The EB theoretical results were evaluated from the Norros equation [20]. We examined two different CAC mechanisms: i) a descriptor-based CAC (DBCAC) mechanism and ii) a measurement-based $\mathrm{CAC}$ (MBCAC) mechanism. Both $\mathrm{CAC}$ approaches were proposed to deal with time-dependent video sources. The main limitation of the DBCAC mechanisms is that the EB calculus is based on the traffic descriptors. Declaration errors lead to incorrect CAC decisions. MBCAC mechanisms [7.9] that estimate real network resources and parameters were proposed to avoid these limitations and so, ensure accurate CAC decisions.

We propose a new MBCAC for streams with timedependence or scaling characteristics. Consequently. for this the MBCAC the $\mathrm{EB}$ is dynamically re-evaluated using the measured parameters including the time-dependence or Hurst degree. The AV [26.27] estimator was added to the MBCAC mechanism to provide the on-line Hurst estimation. We de-

This paper was presented in part at the 17 th Internarional Teletraffic Congress (ITC). December 200] [23]. 
note this on-line implementation of $\mathrm{AV}$ estimator as $A V_{R T}$ (real time $\mathrm{AV}$ estimator). Hence. for the $\mathrm{MBCAC}$ the $\mathrm{EB}$ results are continuously changing. This is also function of the connections input/output process and the available network ressources.

The acceptance regions were obtained for video conneclions where the scaling was modeled by a fractional Brownian motion [19] (fBm) process and also for real MPEG-1 sequences. The $\mathrm{BBm}$ choice is explained by the fact that it is the only stochastic process that is able to represent the whole scaling degree rangc $(0<H<1)$. Moreover. $\mathrm{fBm}$ is known as the unique gaussian H-sssi, i.e., self-similar with self-similarity parameter and stationary increments $[18.21]$. The $\mathrm{M} / \mathrm{G} / \infty$ model proposed in [14] has a restricted scaling representation. I generates samples with autocorrelation function defined by $e^{-3 \sqrt{k}}$ besides the model remains SRD. The fractional Gaussian noise ( $\mathrm{fGn}$ ) is the $\mathrm{fBm}$ increment process and it captures only LRD $\left(H>\frac{1}{2}\right)$. Furthermore. it is not able to well represent SRD connections [17]. The EB and admission regions results are presented for arrival processes with different scaling degrees $(0<H<1)$ and huffer sizes. A set of simulations were performed to verify the analytical results. We also presented the bounds of the Norros EB equation to deal with video traffic.

The rest of this paper is organized as follows. Section 2, describes the scaling model for representing the timcdependence of the video sequences and the EB evaluation. Section 3 briefly presents the Hurst parameter estimators examined on this work. Section + show a description of the proposed MBCAC mechanism including the on-line estimator of the Hurst parameter. In Section 5 the main results concerning the Hurst estimation. EB. DBCAC and MBCAC regions are presented and discussed. Finally. Section 6 is devoled to the conclusions of this work.

\section{THE SCALING MODEL AND THE EB EVALUATION}

The scaling or dependence degree is here represented by the Hurst parameter. The traffic scaling evolution can be observed by the asymptotic behavior of its correlation function $\left(\rho\left(k^{\circ}\right)\right)$ where $\rho(k) \sim L\left(k^{\circ}\right) k^{2(H-1)}$ and $L(k)=H(2 H-1)$. For traffic with LRD characteristics we have $\sum_{k=-x}^{x} \rho(k)=$ $x$. For anti-persistent or negative dependent processes $(0<$ $H<\frac{1}{2}$ ) we have $\sum_{k=-x}^{x} \rho(k)=0$. And, for SRD processes ( $H=\frac{1}{2}$ ) the correlation function behavior is such that $\sum_{k=-x}^{x} \rho(k)^{2}=c$. where $c>0$ is a finite constant (e.g. pure Brownian motion and Poisson processes.). For traffic engineeering the term SRD is related to the exponential decaying characteristic of the autocorrelation function of the Markov models. Some authors denote all processes with $H \leq \frac{1}{2}$ as SRD.

In this work, the video sequences scaling was modeled by the $\mathrm{fBm}$ stochastic process. The $\mathrm{fBm}$ is a family of Gaussian random variables $\left(X_{H}(t)\right)$ indexed in $\Re$ with zero mean and -ontinuous sample paths (null at time 0 ). The variance of its independent increments is proportional to its time intervals accordingly to the expression

$$
\operatorname{Tar}\left[X\left(t_{2}\right)-X\left(t_{1}\right)\right] \times\left|t_{2}-t_{1}\right|^{2 H} .
$$

for $0 \leq t_{1} \leq t_{2} \leq 1 . \mathrm{Y}_{H}(t)$ is considered a self-similar process if its statistical characteristics ${ }^{2}$ hold for any time scale. Thus, for any $t_{0}$ and $r>0$ we have

$$
\left[X_{H}(t+\tau)-X_{H}(t)\right]_{\tau \geq 0} \stackrel{d}{=} r^{-H}\left[X_{H}(t+r \tau)-X_{H}(t)\right]_{\tau \geq 0}
$$

where the increments are stationary, self-similar and $r$ is the process re-scaling factor. As we note. the characteristics of the $\mathrm{X}_{H}(t)$ process varies according to the $H$ values. Norros [20] proposed a discretization procedure of a $1 \mathrm{Bm}$ process to represent a traffic stream with scaling characteristics. Denote $A(t)$ as the number of received packets by a multiplexer up to time $t$. We have.

$$
A(t)=m t+\sqrt{\operatorname{ain}} X_{H}(t)
$$

where $m$ is the mean rate of the arrival process and $a=$ $\frac{\operatorname{lar}[A(l)]}{\left(n t^{2} \|\right)}$ denotes the variance coefficient also known as peakedness. In this paper. we considered $A(t)$ as one of the arrival process representing a video connection. For the simulation experiments the fBm sample paths were generated by the well-known random midpoint displacement (RMD) [2] algorithm. In [10] other traffic models are investigated 10 represent video arrival processes with different scaling characteristics.

\subsection{THE EFFECTIVE BANDWIDTH EVALUATION}

Consider a queue system with deterministic service and infinite buffer $Q$. Norros [20] proposed an analytical approximation to evaluate the required bandwidth $C_{A}(n)$. of $n$ homogeneous $1 \mathrm{Bm}$ sources. For a delailed description of EB definitions and formulations the reader should refer to [12]. Suppose that each connection has mean $(m)$. variance $(a)$ and dependence degree $(H)$ parameters. If $\epsilon=P(Q>B)$ is the probability that a buffer of size $Q$ becomes larger than a limil $B$, the required bandwidth $C_{A}(n)$ for an aggregate of $n$ connections is defined as

$$
\begin{aligned}
C_{A}(n)= & n m+(\kappa(H) \sqrt{-2 \ln \epsilon})^{1 / H} \\
& \cdot B^{-(1-H) / H}(n n a)^{1 /(2 H)}
\end{aligned}
$$

where $r(H)=H^{H}(1-H)^{1-H}$. Hence, the EB of each connection is

$$
C_{e}(n)=\frac{C_{A}(n)}{n} .
$$

Suppose that $n$ connections were previously admitted in a link with capacity $C$. When a new connection requesi is received, the EB for this new connection $(n+1)$ is evaluated by $C_{\epsilon}(n+1)=C_{A}(n+1) /(n+1)$. The new connection is accepted by the CAC only if $C_{C}(n+1)<C-C_{A}(n)$. Otherwise. the connection request is rejected.

? By statistical diaracteristics. we mean marginal distlibution and dependence degree. Only in this case a process should be considered as selfsinilar. 


\section{HURST PARAMETER ESTIMATION}

To determine the impact of the scaling on the admission regions we need accurate Hurst estimation. Hence. we should examine different Hurst estimators. A brief description of the the main estimators included in the $\mathrm{HEP}^{3}$ is next presented. They are the R/S (Rescaled Adjusted Range) statistic, the Higuchi and the wavelet-based Abry-Veitch (AV) estimators.

\subsection{R/S STATISTICS}

Consider a sequence of random samples $\left\{X_{i}\right\}$ with partial sums $Y(n)=\sum_{i=1}^{\prime \prime} X_{i}$ and sample variance $S^{2}(n)=$ $\cdot 1 / n) \sum_{i=1}^{n} X_{i}^{2}-(1 / n)^{2} Y^{2}(n)^{2}$. The R/S statistics is given by

$$
\begin{aligned}
\frac{R(n)}{S(n)}= & \frac{1}{S(n)}\left[\max _{0 \leq t \leq n}\left(Y(t)-\left(\frac{t}{n}\right) Y(n)\right)\right. \\
& \left.-\operatorname{mini}_{0 \leq t \leq n}\left(Y(t)-\left(\frac{t}{n}\right) Y^{\top}(n)\right)\right] .
\end{aligned}
$$

We have that $E[R(n) / S(n)] \sim C_{\mathrm{I}} n^{H}$ for $n-\infty$, where is a positive constant. The Hurst parametel is then ob-ained by a linear regression in a log-log plot of $R(n) / S(n)$ ersus $n$. The main advantage of the $R / S$ statistics is its inzependence from the stream marginal distribution under estimation.

\subsection{HIGUCHI ESTIMATOR}

The Higuchi estimator is based on the fractal dimension ${ }^{+}$ $\supset$ of the time series. Consider a random sequence $\left\{X_{i}\right\}, i=$ $\ldots . . . m$ with partial sums, $Y(m)=X_{1}+X_{2}+\ldots+X_{m}$. We can get the following sample sequences

$$
\begin{gathered}
Z_{k}^{m=}=\{Y(m) \cdot Y(m+k) \cdot Y(m+2 k) \ldots . \\
\left.Y\left(m+\left\lfloor\frac{N-m}{k}\right\rfloor k\right)\right\}
\end{gathered}
$$

where $k=1.2 \ldots N, m=1.2 \ldots k$. and the operator $x$ stands for the largest integer smaller than $x$. For each jequence $Z_{l}^{m}$. we evaluate the normalized curve length

$$
\begin{aligned}
L_{m}(k)= & \frac{N-1}{k^{2}\left\lfloor\frac{N-n_{1}}{k}\right\rfloor} \sum_{i=1}^{\left\lfloor\frac{N-m}{h}\right\rfloor} \mid Y(m+i k) \\
& -I^{\prime}(m+(i-1) k) \mid
\end{aligned}
$$

and define the curve length $L\left(k_{i}\right)$ for each lag $k_{i}$ as

$$
L(k)=\frac{1}{k} \sum_{m=1}^{h_{i}} L_{m}\left(k_{i}\right) .
$$

Hence. $E[L(k)] \sim C_{2} k^{-D}$ for $k \rightarrow \infty$, where $D=2-H$. The $H$ parameter is then estimated by regression in a plot $\log L(k)$ by $\log (h)$.

TTo obtain the HEP software send an e-mail to coelhorime. eb. Dr.

${ }^{4}$ A derailed definition of tractal dimension can be found in [3]. Chapter V. pp.171-201.

\subsection{ABRY-VEITCH ESTIMATOR}

The AV estimator ${ }^{5}$ uses the discrete wavelel transform (DWT) to successively decompose a sequence of samples in approximation $\left(a\left(j, k_{i}\right)\right)$ and detail $\left(d\left(j, k^{\prime}\right)\right)$ coefficients. These coefficients are indexed by its decomposition scale $j$ and time $k$. The authors showed the relation between these delail coefficients and the Hurst parameter. The AV estimation can be described in three phases:

1. Wavelet decomposition: the DWT is applied to the sample data generating the detail coefficients $d(j . k)$.

2. Variance estimation of the detail coefficients: for each scale $j$. we evaluate the variance $\mu_{j}=$ $\left(1 / n_{j}\right) \sum_{k} d(j \cdot k)^{2}$. where $n_{j}$ is the number of available coefficients for each scale $j$.

3. Hurst parameter estimation: we plot $y_{j}=\log _{2}\left(\mu_{j}\right)$ by $j$. Using a weighted linear regression. we get the slope $a$ of the plot and the $H$ parameter is estimated as $H=$ $(1+a) / 2$.

The AV estimator is robust to polynomial trends added to the process under analysis. Moreover. the AV is appropriate for real time estination [27] and so. to deal with transient situation. This real time estimator $\left(A T_{R T}\right)$ was included in the proposed MBCAC mechanism presented in Section 4.

\section{MBCAC FOR TIME-DEPENDENT VIDEO CONNECTIONS}

The proposed MBCAC is based on a previous work presented in [7]. In this earlier approach a Kalman filter was used to achieve the mean and variance estimation of the aggregated traffic. In our proposal, we included the $A T_{R T}$ estimator and also an algorithm that dynamically evaluates the $\mathrm{EB}$ of the connections using the measured $(m . \imath . H)$ parameters. Figure 1 illustrates the proposed hybrid MBCAC mechanism.

The EB evaluation for the time-dependent streams was determined as presented in Section 2.1. The main difference is that the EB is now evaluated with the real-time estimated parameters. In our proposition, the EB is re-evaluated only when a connection leaves the network. This procedure allows faster decisions by the CAC mechanisms.

The measured mean and variance of the aggregated trafific represents a state vector $X_{k}$ of the Kalman filter. A state changing occurs each time a connection enters or leaves the network. For each state. $k$, the Kalman filter stage gives an estimation of the mean $M_{k}$ and variance $V_{k}$ of the aggregated traffic. The main function of the Kalman filter is to provide a weighted estimation between the declared and the measured paramelers [7].

The $A I_{R T}$ estimator is implemented by a filter bank as depicted in Figure 2. The filter bank is a cascade of low-pass and band-pass digital filters followed by decimators. The output of a low-pass filter is injected on a new pair of filters generating the approximation $a\left(j . k^{2}\right)$ and detail $d(j, k)$ coefficients. As shown in Section 3. the Hurst parameter is 
New

connections

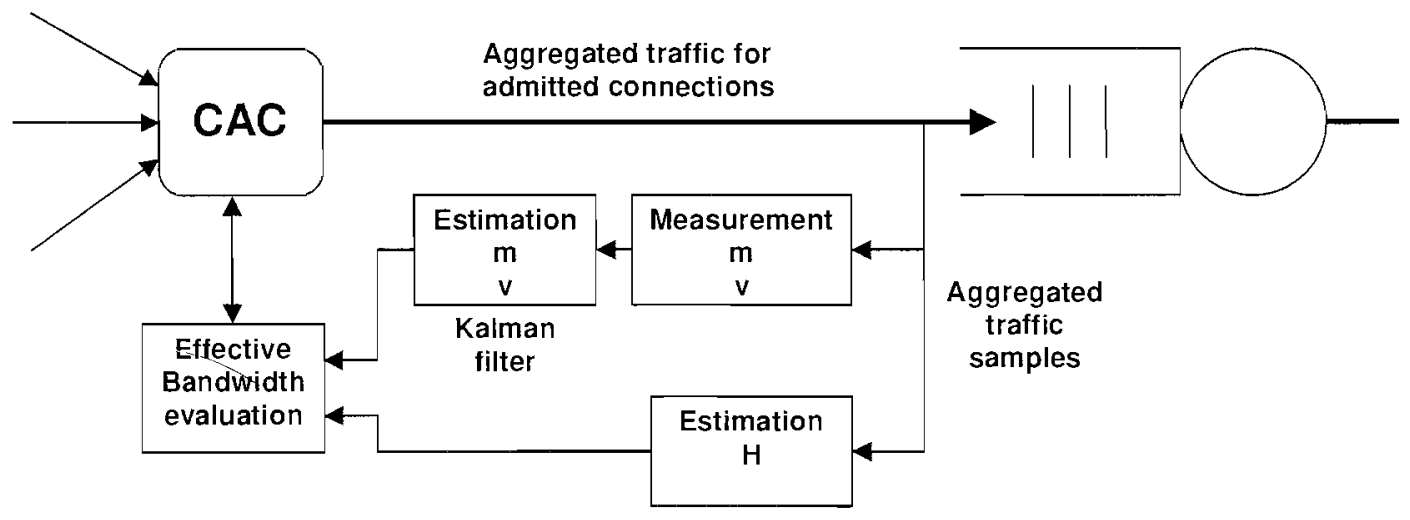

Figure 1. General diagram of the proposed hybrid MBCAC.

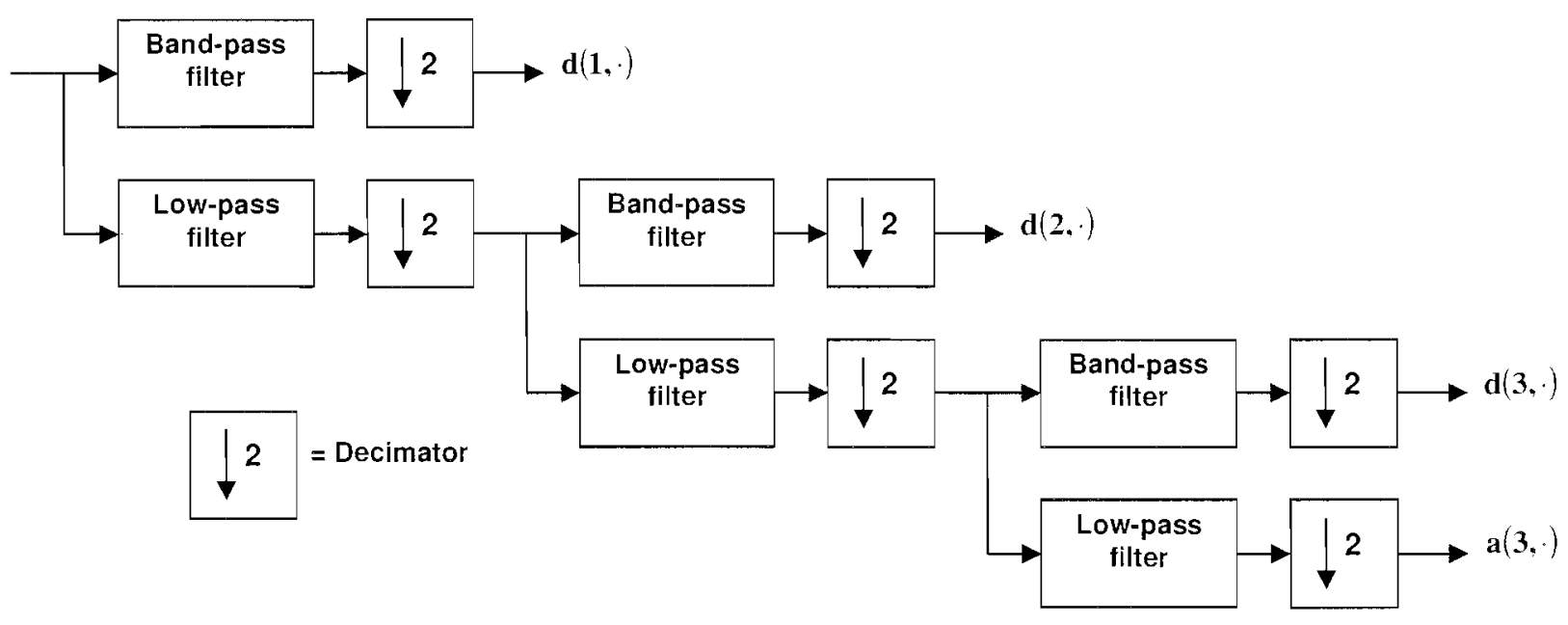

Figure 2. Filter bank stages for the $A T_{R T}$ estimator.

obtained from the detail coefficients $d(j . k)$. The main difference now is that the $\mu_{j}$ parameters are updated in realtime. We need to keep two parameters for each scale. the $\operatorname{sum} S_{j}=\sum_{k=1}^{n_{j}} d^{2}(j, k)$ and the number of generated detail coefficients $n_{j}$. For each new detail coefficient $d(j . k)$. these values are updated as $n_{j}-n_{j}+1$ and $S_{j} \leftarrow S_{j}+d^{2}(j \cdot k)$. The $\mu_{j}$ parameters are re-evaluated at any time where $\mu_{j}=$ $S_{j} / n_{j}$. These $\mu_{j}$ values enables the weighted regression to obtain the Hurst estimation. The input traffic samples $A(t)$. are obtained at constant sampling intervals $t_{m}$. The choice of this sampling interval is very important to the measurement process. In our investigation we adopted the definition presented in [13]. The sampling interval must be in the range $d_{\max }<t_{m}<200 d_{\max }$ where $d_{\max }$ is the largest delay in the system. given by $d_{\max }=B / C$. These are the timescales of interest for a measurement process in a queue-server system with capacity $C$ and finite buffer $B$.

\section{RESULTS AND DISCUSSIONS}

In this section we present the main theoretical and simulations results. These results conccrns the scaling or H estima- tion (Section 5.2). the EB and DBCAC obtained regions (Section 5.3) and finally, the MBCAC admission regions (Section 5.4).

\subsection{ANALYSIS ENVIRONMENT}

To evaluate the admission regions for the DBCAC and MBCAC mechanisms we considered the real video sequences Table-TTennis, Salesman, Bond and Race. The Table-Tennis and Salesman standard sequences [5] were encoded in MPEG-2 and H.261 with sampling rate of 50 frames $/ \mathrm{sec}$ and $360 \mathrm{GOB} / \mathrm{sec}^{6}$, respectively. The Bond and Race sequences [25] were MPEG-1 encoded with sampling rate of 25 frames/sec. The Table-Tennis and Salesman sequences were originally packetized in cells ( 1 cell=124 bits). For simplicity, the original MPEG-1 traces were converted to cells/frame. Table l presents the mean $(m)$ and standard deviation $(\sigma)$ of these sequences. These values are shown for the sampling period of each the sequence. These statistical parameters were considered as traffic descriptors by the DBCAC mechanisn. The $\mathrm{fBm}$ process was generated

\footnotetext{
Each H.261 frame consists of 12 GOBs or group-of-blocks
} 

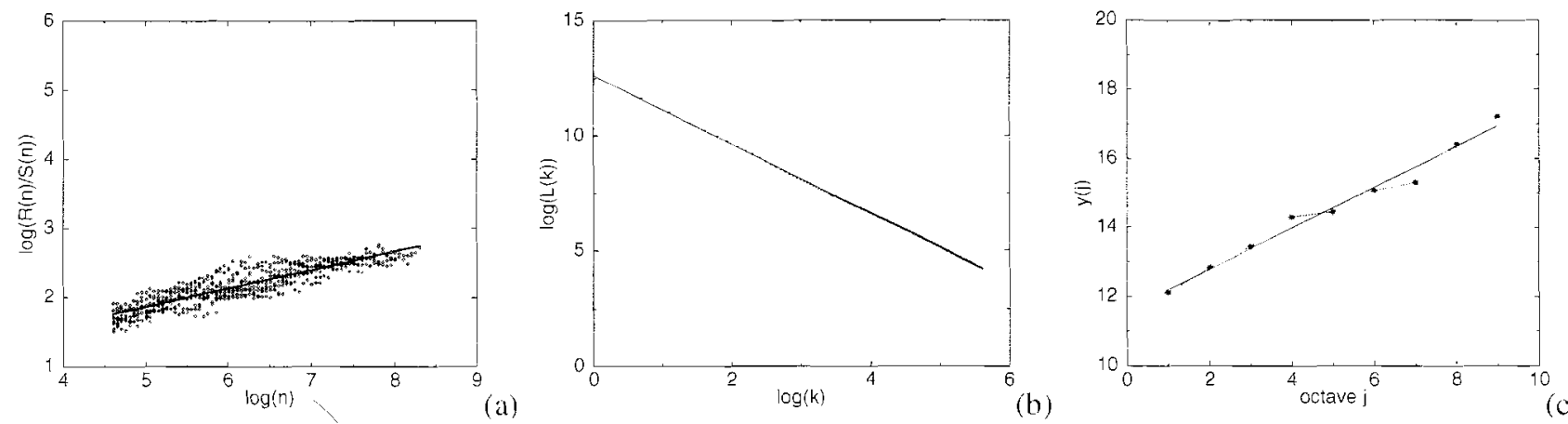

Figure 3. Hurst estimation TTennis sequences with (a) R/S. $H=0.2$ (b) Higuchi. $H=0.5$ (c) AV. $H=0.8$

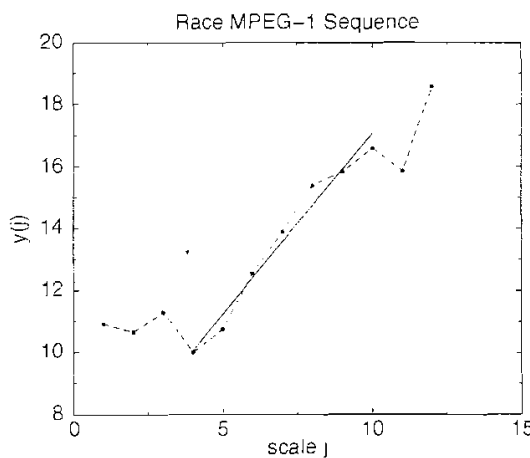

Figure 4. AV log-scale plot for Race sequence

\begin{tabular}{lll}
\hline & $m$ & $\sigma$ \\
\hline Table-Tennis & 480 cells/frame & 96.7 \\
Salesnan & 3.258 cells/GOB & 0.631 \\
Bond & 63.3 cells/frame & 66.9 \\
Race & 170.27 cells/frame & 50.74 \\
\hline
\end{tabular}

Table 1. Parameters of the video sequences.

considering the $m$ and $\sigma$ parameters obtained from the real Table-Tennis and Salesman sequences. These fBm sequences have also different $H$ values, e.g. $H=0.2, H=0.5$ and $H=0.8$. These three $H$ values represent streams with antipersistence, SRD and LRD. respectively. For the theoretical admission regions evaluation we considered the upperbound linear approximation.

\subsection{HURST ESTIMATION RESLILTS}

As mentioned before, to examined the impact of the video sequences scaling on the admission regions we need an accurate Hurst estimation. Thus, in this section we show the results of the video connections olf-line scaling estimation for the R/S. Higuchi and AV methods (see Table 2). These $H$ results are considered for the $\mathrm{EB}$ evaluation and the $\mathrm{DB}$ CAC regions. Figure 3 also depicts an example of a Hurst estimation of the TTemis sequence of each method.

From Table 2 we note the importance of using different estimators 10 obtain an accurate scaling characterization. These estimators were concieved based on different assumptions

\begin{tabular}{llll}
\hline & $\hat{H}(\mathrm{R} / \mathrm{S})$ & $\hat{H}$ (Higuchi) & $\hat{H}(\mathrm{AV})$ \\
\hline TTennis02 & 0.26 & 0.21 & 0.18 \\
TTennis05 & 0.51 & 0.50 & 0.54 \\
TTemnis08 & 0.81 & 0.73 & 0.85 \\
Salesman02 & 0.29 & 0.25 & 0.20 \\
Salesman05 & 0.53 & 0.47 & 0.46 \\
Salesman08 & 0.81 & 0.78 & 0.75 \\
Bond & 0.96 & 0.89 & 1.10 \\
Bond & 0.80 & 0.67 & 1.09 \\
\hline
\end{tabular}

Table 2. Hurst parameter estimation results.

concerning the samples distribution. We can see that the estimators presented similar results for the sequences generated from the $\mathrm{fBn}$ process. However, there is a difference in the estimation results for the real MPEG-1 sequences (Bond and Race). This fact can be explained by the high scaling variability. the short-lime duration and non-gaussian distribution of these sequences. The scale choice of an AV off-line estimation is more complex when dealing with samples with high scaling variability ou multi-scaling characteristics. An example of this scale choice complexity is illustrated in Figure 4 for the Race sequence. In Section 5.4, we will see that the $A V_{R T}$ achieved better accurate results for on-line or continuous estimation.

An incorrect estimators choice leads to erroneous traffic scaling interpretation and so. CAC decisions. This reinforces the idea that different estimators should be used to decide the 

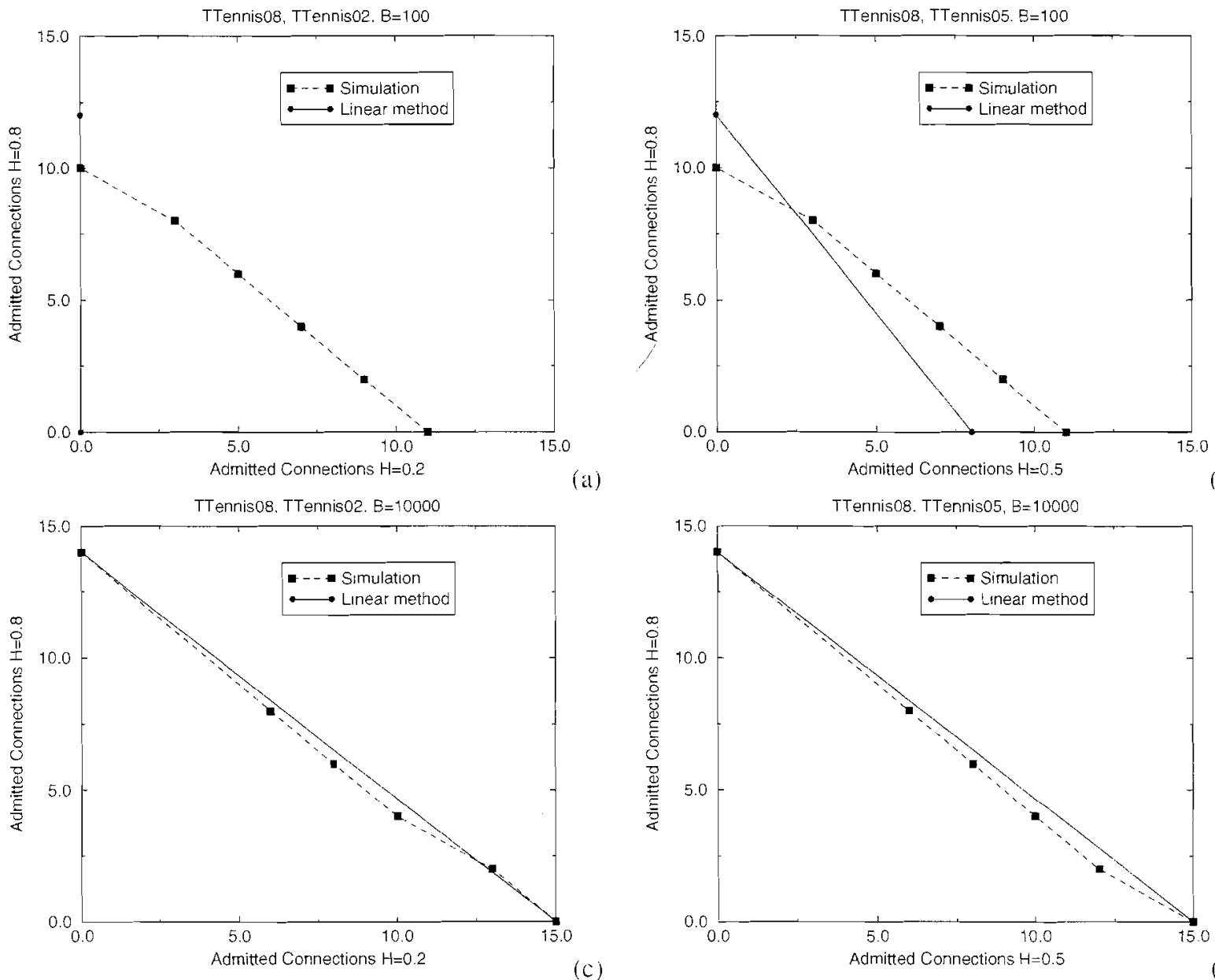

(b)

Figure 5. Admission regions (a) (b) buffer $=100$ cells (c) (d) buffer $=10000$ cells.

scaling degree to be considered by the CAC mechanism. In this work we adopted the R/S $H$ values since we had similar results for the $\mathrm{fBm}$ sequences for all estimators. Moreover, for the real MPEG-1 Bond and Bond sequences the Higuchi and $\mathrm{AV}$ methods did not present accurate results. If no knowledge about the samples distribution is available we should use an average Hurst value from the obtained results considering a certain confidence interval.

\subsection{EB AND DBCAC REGIONS RESULTS}

Several simulations were run to validate the EB theoretical results (see Eq. 3) and the DBCAC acceptance regions. For the EB analysis we considered that the video sources were multiplexed in a finite queue with deterministic service ( $C=155 \mathrm{Mbps}$ ) to obtain the maximum number of calls considering a $C L R=10^{-4}(\approx \epsilon=P(Q>B))$. The analytical and simulation results are presented in Table 3 as a function of different buffer sizes ( $B$ in cells). The TTemmis05 ( $H=1 / 2$ ) sequence is representing a SRD traffic stream. As we observe (see Table 3 ), the analytical results were valid only for $H>1 / 2$ and $B>5000$. i.e. large buffer sizes. Thus, from these values we considered that these are the bounds $(H>1 / 2$ and $B>5000)$ for the Norros equation when dealing with $\mathrm{fBm}$ video traffic and different $H$ values. Traffic sources with $H \leq 1 / 2$, present-

\begin{tabular}{lccccc}
\hline & \multicolumn{5}{c}{ Buffer Size } \\
\hline & 100 & 500 & 1000 & 5000 & 10000 \\
\hline TTemnis02 & $0-11$ & $8-15$ & $1.3-15$ & $15-15$ & $1.5-15$ \\
TTennis05 & $8-11$ & $12-13$ & $13-14$ & $14-15$ & $15-15$ \\
TTemiso8 & $12-10$ & $12-11$ & $13-12$ & $13-13$ & $14-14$ \\
\hline
\end{tabular}

Table 3. Number of adnitted connections-analytical \& simulation results.

ing the anti-persistence effect or negative correlation $[4,16]$. are strongly centered around the mean ratc. Generally, this anti-persistent traffic presents better queueing performance compared to sources with LRD characteristic. Moreover. small buffer is a major requirement when considering realtime video applications. Hence. a new EB equation should be investigated for $H<1 / 2$ and small buffers. Figure 5 illustrates the DBCAC admission regions obtained by the upperbound linear ${ }^{7}$ method considering different buffer sizes.

As we observe, the admission regions for LRD connections are close to SRD connections cven for large buffer sizes ( $B=10000$ cells), as shown in Figures 5.c and 5.d. These

TThe linear method is denoted by CAC procedures as an upperbound since in achieves the most optimistic regions where multiplexing is only con sidered among a connection type. i.e.. identical traffic sources 


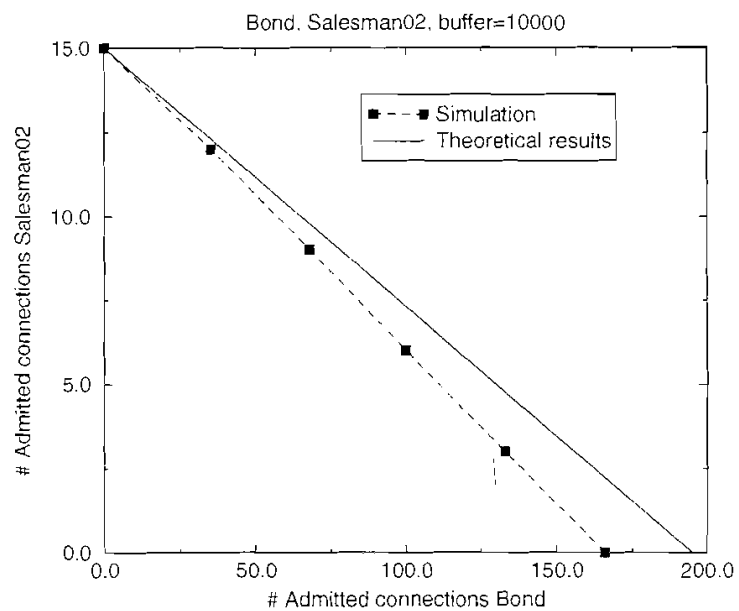

Figure 6. Admission regions for connections with different marginal distributions.
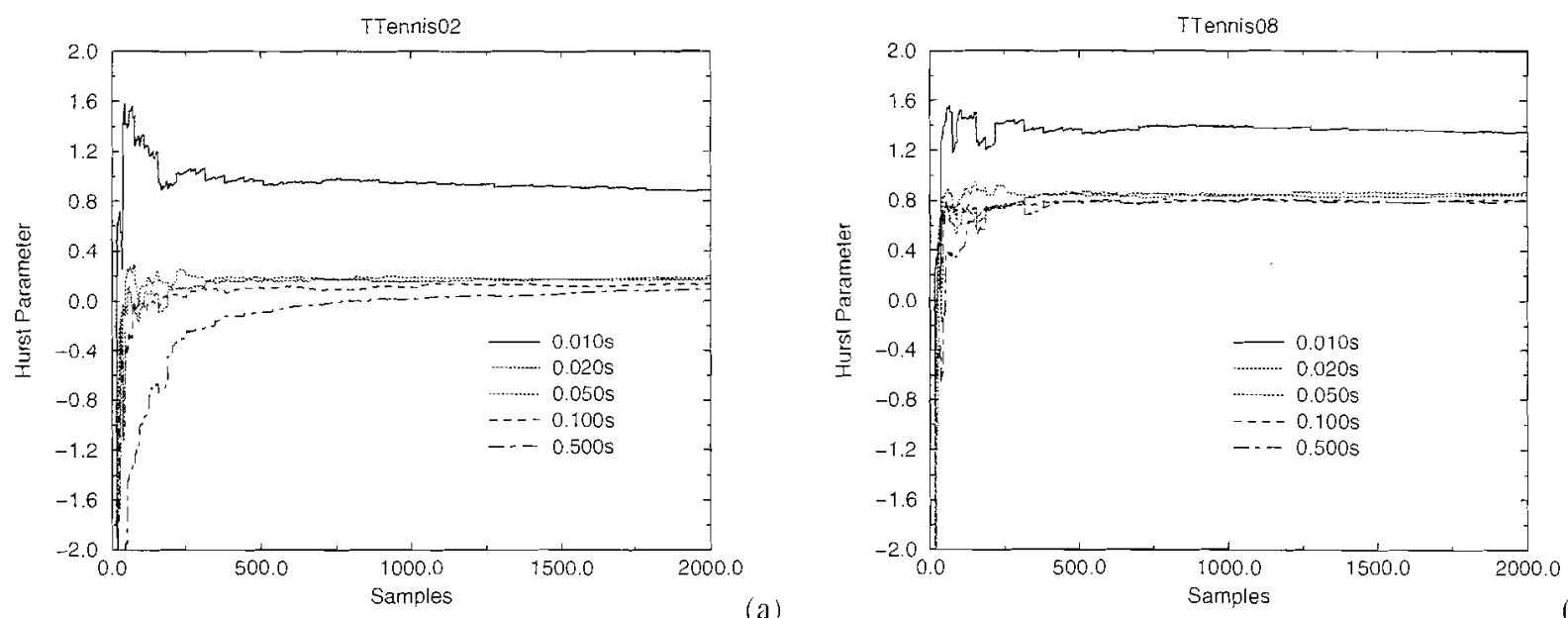

(a)

Figure 7. On-line Hurst estimation with different $t_{m}$ values (a) TTennisO2 sequence. (b) TTennisO8 sequence.

results confirm the conclusions presented by Ryu [28] where he showed that LRD has no significant impact on network performance. However. this does not mean that the scaling characteristics has no importance for traffic engineering. For exemple. in [30] the authors showed that the scaling degree knowledge can improve the performance of rate-based feedback mechanisms. We extended the analysis for the real MPEG-1 Bond sequence. The main objective was to verify the impact of other distribution. i.e.. non-gaussian, on the admission regions. Figure 6 shows the analytical (Linear method) and simulation results for SalesmanO2 and Bond sources. considering $C=15.5 \mathrm{Mbps}$ and $B=1000$ cells. As we previously observed, the regions were similar when considering only the fBm sources. Here, however. the admission regions were more sensible to the traffic distribution than the scaling degree even for large buffers. We note that when the number of Bond sources increases this difference becomes larger. This means that the marginal distribution was responsible for the impact on the number of admitted connections. In an ongoing paper [10] we show that compared to scaling characteristics the video heavy-tail distribution has greater impact on network performance and so admission regions. In [1] the authors proposed a markovian model that represents the dependence degree over four or five time scales. A further research should investigate if these time scales are sufficient to enable the analysis of the impact of the dependence degree on $\mathrm{CAC}$ regions.

\subsection{MBCAC AND ON-LINE SCALING ESTIMATION RESULTS}

To examine the performance of the MBCAC mechanism, we implemented a connection input/output algorithm. We assume a constant time interval $T_{C}$ between transitions i.e.. arrival and departure of a connection. When a connection leaves the notwork. the EB is re-evaluated based on the estimated parameters $\left(\hat{m}_{k} . \hat{r}_{k} \cdot \hat{H}_{k}\right)$. Then. the maximum number of admitted connections is computed.

We inserted an error in the mean and variance declared deseriptors to verify the MBCAC performance. We considered that each comection declares parameters $20 \%$ bellow its real values, e.g. real mean $m=480$ eells/frame and declared mean $m_{d}=38 \pm$ cells/frame for the TTennis sequence.

Figure 7 depicts the on-line estimated results of the Hurst parameter when using the $A T_{R T}$ estimator for the the TTenmis02 and TTennis08 sequences. The curves indicate an ini- 

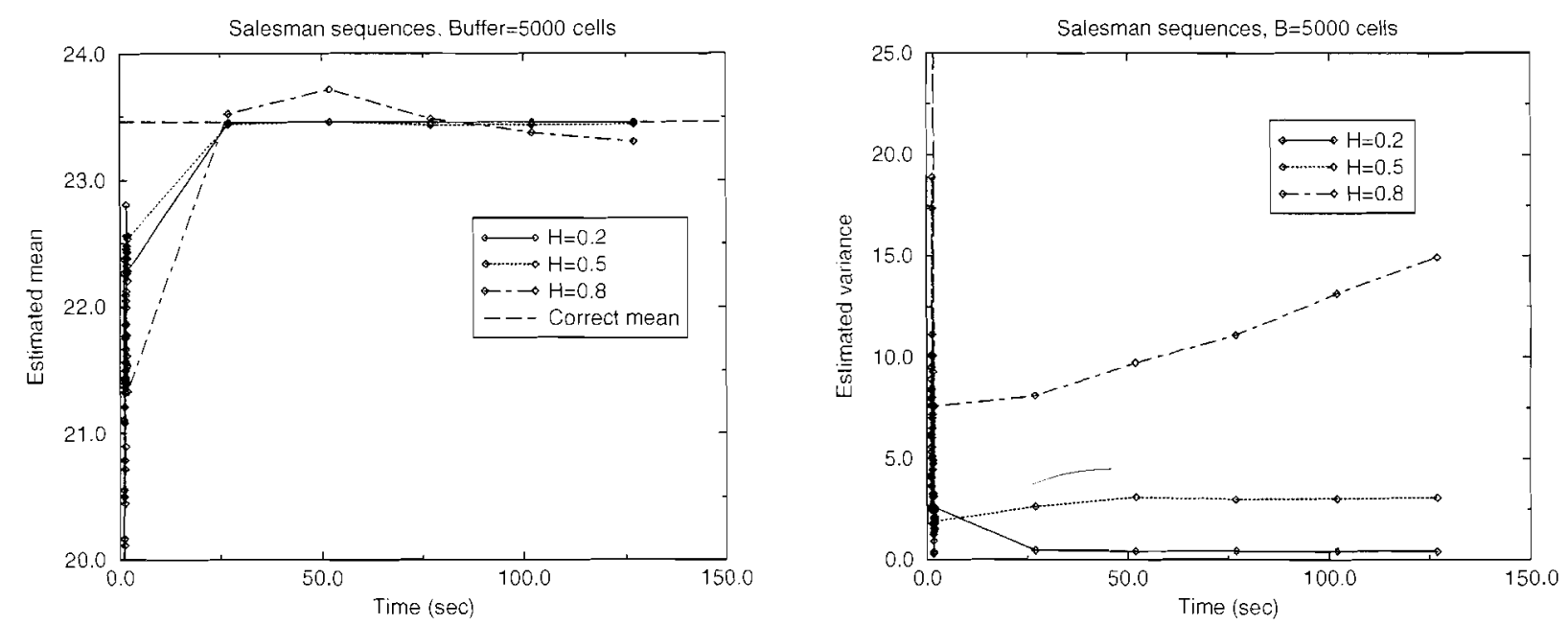

Figure 8. (a) Mean (h) variance estimation in MBCAC mechanism.
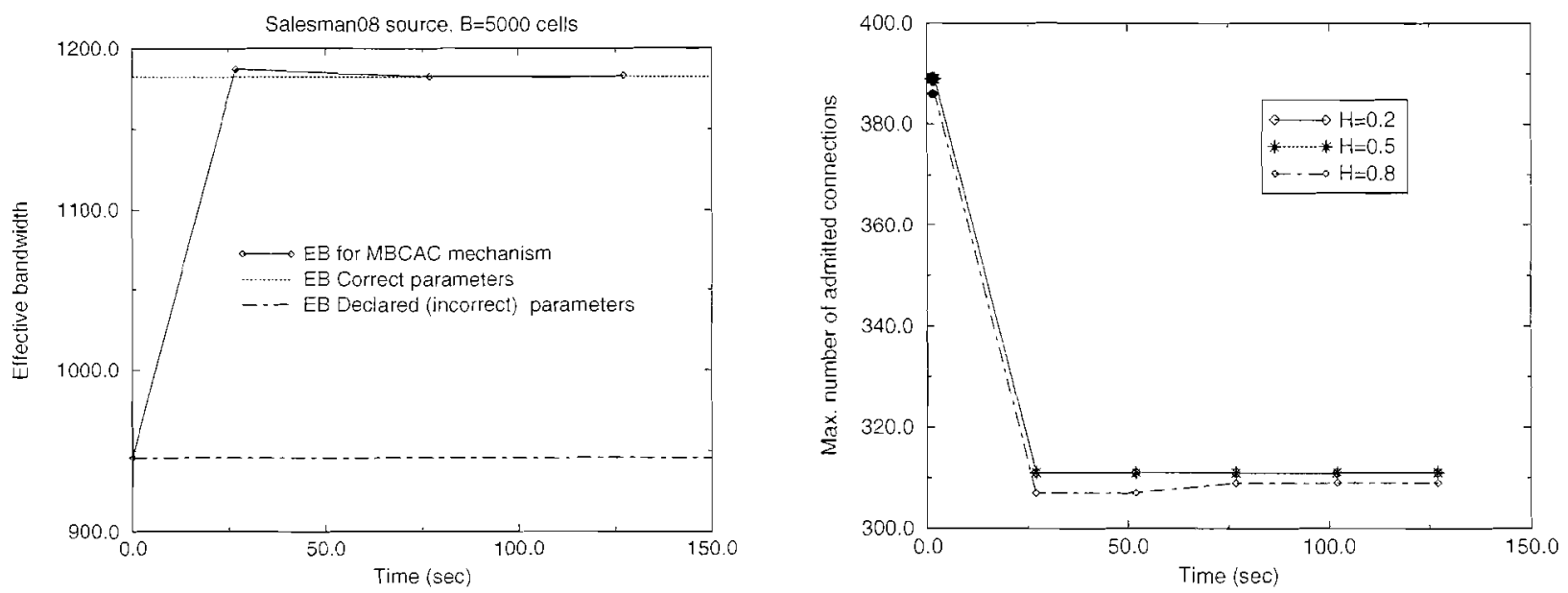

Figure 9. (a) Effective bandwidth (b) the max, number of connections for different $H$ values.

tial transient period after which the $A I_{R T}$ converges to the original Hurst value. The choice of the $t_{m}$ is very important for the accuracy of the Hurst estimation. We considered $t_{m}$ values of $0.01 \mathrm{~s} .0 .02 \mathrm{~s} .0 .05 \mathrm{~s} .0 .1 \mathrm{~s}$ and $0.5 \mathrm{~s}$. As we note, $t_{m}$ values $<0.02 \mathrm{~s}$ lead to incorrect estimation for both LRD and SRD sequences. In [26] Roughan and Veitch also prove that Hurst values can present high scaling variability over time. The presence of this multi-scaling behavior on network traffic is discussed in [22]. Hence, the Hurst value should not be considered as constant for a long period of time. Only a realtime estimation is able to detect this multi-scaling variation. This variation can change the EB results and so, the admission regions. The $A T_{R T}$ also showed good performance for individual source Hurst estimation when considering the input/output of other video connections.

The individual mean and variance measurements are depicted in Figure 8 for the Salesman sequences with different Hurst values. The curves were obtained from a simulation set of 20 homogeneous sources, $T_{c}=2.5 \mathrm{~s}$ and $t_{m}=0.02 \mathrm{~s}$. The curves presenting the mean measurements (Figure 8.a) show a slower convergence for LRD sources. For all cases, the MBCAC mechanism detected the declaration errors and the estimation achieved the correct mean value. The variance curve (Figure 8.b) indicates the aggregation effect of connections with different dependence degrees. For anti-persistent traffic, the variance decreases with the video connections aggregation.

This smoothing characteristic of the anti-persistent $(H<$ $\left.\frac{1}{2}\right)$ traffic is very interesting for network performance and buffer dimensioning. In the case of SRD traffic $\left(H=\frac{1}{2}\right)$. the variance is constant and similar to the original value (Figure 8.b). For LRD traffic, the variance increased with the aggregation of connections but no impact was detected due to this variance increasing. This agreggation effect is detected by the MBCAC mechanism. Once more, it is demonstrated the importance of measurement based procedures usage.

Figure 9.a presents the results obtained by the EB stage with the estimated parameters of the MBCAC for a LRD Salesman sequence. The EB results are continuously updated as the measurements are obtained from the MBCAC. The maximum number of admitted connections considering the EB results is presented in Figure 9.h. A high number of connections $(\approx 390$ ) could be admitted by the CAC mechanism if the $\mathrm{EB}$ is based on traffic descriptors. However, when $\mathrm{EB}$ 


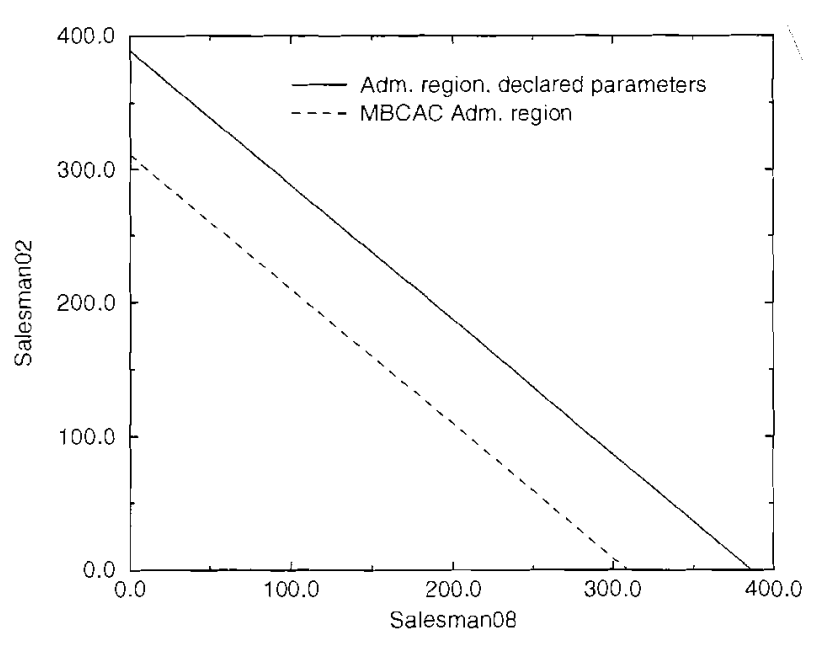

Figure 10. Admission regions for declared and estimated parameters.

is re-evaluated. i.e.. using the measurement parameters, the admission region changes roughly. Another interesting result reinforces that the number of admitted connections were very close despite of the dependence degree.

Admission regions for declared and estimated parameters are shown in Figure 10 for different Salesman sequences. As we see, traditional DBCAC mechanisms are not able to detect declaration errors leading 10 wrong CAC decisions. This proves that broadband packet networks should adopt measurement based procedures for traffic with scaling characterislics.

\section{CONCLUSIONS}

The impact of the time-dependence or scaling degree on video admission regions has been evaluated in this paper. We investigated two different CAC approaches. The first approach was based on a DBCAC mechanism where the $\mathrm{EB}$ evaluation was based on the video traffic descriptors. A secand approach named MBCAC was proposed to deal with network measured parameters including an on-line scaling degree estimator. This on-line Hurst estimation improved the $\mathrm{EB}$ accuracy and hence the CAC decisions.

The off-line Hurst estimation results have demonstrated that the R/S estimator is accurate and robust for processes sith any marginal distribution. We also showed that only an on-line estimation is able to detect the traffic scaling variation.

This study has also showed that the dependence or scaling degree has no significant impact on the admission regions. Furthermore, we noticed that the traffic marginal distribution ?rovoke more impact on the $\mathrm{CAC}$ regions.

Further research should include markovian models that represents the scaling degree during some scales as well as te use of the scaling degree knowlegde to improve network control performance.

\section{ACKNOWLEDGEMENTS}

This work was supported by Faperj under the grant number E26-151073/99.

\section{REFERENCES}

[1] A. Andersen and B. Nielsen. A markovian approach for modeling packet traffic with long-range dependence. IEEE JSAC, 16(5):719-732. lune 1998.

[2] M. Barnsley. The Science of Fractal Images. Springer-Verlag Inc. New York. USA, 1988.

[3] M. Barnsley. Fractals Everymhere-2nd Edition. Academic Press. 1993.

[4] R. Coelho and L. Decreusefond. Statistical performance of $1 v /$ hdt traffic over broadhand digital networks. In IEEE GLOBECOM 98 . November 1998.

[5] R. Coelho and $S$. Tohmé. A generic smoothing algorithm for real time variable bit rate video traffic. Computer Networks and ISDN Systems. 29(7):2053-2066. Fehruary 1998.

[6] M. Crovella and A. Bestravos. Explaining world wide web traffic self-similarity. In ACM SIGMETRICS'96. May 1996.

[7] Z. Dziong, M. Juda, and L. Mason. A framework for bandwidth management in ATM nelworks - aggregale equivalcnt bandwidth estimation approach. IEEE/ACM Transactions on Newrorking. 5(1):134-147. February 1997.

[8] M. Garret and W. Willinger. Analysis. modeling and generalion of self-similar vbr video trafific. In ACM SIGCOMM 94. London. September 1994

[9] M. Grossglauser and D. Tse. A framework for robusi measurement-based admission control. IEEE/ACM Transations on Netrorking. 7(3):293-309. June 1999.

[10] A. Guimarães and R. Coelho. Video traffic models performance with restricled and non-restricled scaling characterization. To appear in the International Telecommunitions Simposium-ITS2002, September 2002.

[11] H. E. Hurst. Long-term storage capacity of reservoirs. Transactions of American Society of Civil Engineers, 116:770-799. 1951.

[12] F. Kelly. Notes on effective bandwidths. In F. P. Kelly, S. Zachary, and 1. Ziedins. editors. Slochastic Networks: Theory and Applications. volume 4 of Roval Statistical Societs Lecture Notes. pages 141-168. Oxford University Press. 1996.

[13] Y. Kim and S. Li. Timescale of interest in traffic measurement for link bandwidth allocation design. In INFOCOM 96. pages 7.38-748, June 1996.

[14] M. Krunz and A. Makowski. Modeling video traffic using $\mathrm{m} / \mathrm{g} / x$. IEEE JSAC. 16(5):733-748. June 1998.

[15] W. Leland, M. Taqqu. W. Willinger, and D. Wilson. On the self-similar nature of ethernet traffic (extended version). IEEE/ACM Trunsactions on Netrorking. 2(1):1-15. February 1994.

[16] Q. Li and D. L. Mills. Investigating the scaling behavior. crossover and anti-persistence of internet packet delay dynamics. In IEEE Globecom'99, 1999.

[17] S. Ma and C. Ji. Modeling heterogeneous network trafic in wavelet domain. IEEE/ACM Tirnsactions on Networking. 9(5):634-649. Oclober 2001.

[18] B. Mandelbrot and J. Van Ness. Fractional brownian motions. tractional noises and applications. SIAM Review: 10(4):422437. October 1968.

[19] B. B. Mandelbrot and J. W. Van Ness. Fractional brownian motions.fractional noises and applications. SIAM Rer: 10:422437. 1968. 
[20] I. Norros. On the Use of fractional brownian motion in the theory of connectionless network. IEEE Joumal on Selected Areas in Communications, 13(6):953-962. August 1995.

[21] K. Park and W. Willinger. Self-Similar Network Traffic: An Orervien. John Wiley \& Sons, Inc. 2000.

[22] K. Park and W. Willinger. Self-Sinilar Network Traffic and Perfomance Eraluation. Iohn Wilcy \& Sons, Inc. 2000.

[23] R. Pontes and R. Coelho. The scaling characteristics of the video traficic and its impact on acceptance regions. Proceedings of the 77 th Intemational Teleraffic Congress. 4:197-210. December 2001 .

[24] R. Riedi and W. Willinger. Toward an improved understading of network traffic dynamics. Self-Similar Netrork Traffic and Performance Evaluation. John-Wiley \& Sons:507-530. 2000.

[25] O. Rose. Statistical properties of mpeg video traffic and their impact on traffic modeling in atm systems. Proceedings of the 20th Annual Conference on Local Conputer Netrorks, pages 397-406. 1995.

[26] M. Roughan and D. Veitch. A study of the daily variation in the sell-similarity of real data traffic. In 76 th Intemational Teletraffic Congress - ITC 16. pages 67-76. UK. June 1999.

[27] M. Roughan. D. Veith, and P. Abry. Real-time estimation of the parameters of long-range dependence. IEEE/ACM Transactions on Networking. 8(4):467-478. August 2000.

[28] B. Ryu and A. Elwalid. The importance of long-range dependence of vbr video traffic in ATM traftic engineering: Myths and realities. In ACM SIGCOMM 90. 1996.

[29] M. Taqqu. V. Teverovsky, and W. Willinger: Estimators for long-range dependence: An empirical study. Fractals, 3(4):785-788. 1995.

[30] T. Tuan and K. Park. Multiple time scale congestion control for self-similar network traffic. Performance Evaluation, 36:359386. 1999.
Raniery Pontes is a network engineer at the Rede Nacional de Pesquisa (RNP) since 2000. He received his B. degree in Electrical Engineering from the Universidade Federal do Pará (UFPA) in 1996 and his MSc. degree from the Pontifícia Universidade Católica do Rio de Janeiro (PUC-Rio) in 2000. He is currently managing the deployment of multicast in the RNP national hackbone.

Rosângela Coelho is an associate professor at the Electricat Engineering Department of the Instituto Militar de Engenharia do Rio de Janeiro (IME). She received her MSc degree from the Pontifícia Universidade Católica do Rio de Janeiro (PUC-Rio) in 1991 and her $\mathrm{PhD}$ degree from the Ecole Nationale Supérieure des Télècomunications (ENST-Paris) in 1995 both in Electrical Engineering. Her main research interests include traffic modeling, networks and systems performance analysis, fractal modeling and estimation and switched optical networks. 